\title{
SULL'USO DELLE PRO-FORME NELLE RIPRESE COREFERENZIALI DEL TESTO
}

\begin{abstract}
Gałkowski Artur, Sull'uso delle pro-forme nelle riprese coreferenziali del testo [The use of pro-forms in coreferential references of the text], Studia Romanica Posnaniensia, Adam Mickiewicz University Press, Poznań, vol. XXVIl: 2001, pp. 71-82. ISBN 83-232-1039-X, ISSN 0137-2475.
\end{abstract}

The article takes up some aspects of the use of pronouns as referential pro-forms in the process of text substitution. The main emphasis is placed on the distinction between anaphoric and cataphoric pronominalization and on their meaning in coreferential chains. The author states that pronominalization does not occur in all cricumstances but in some cases repetition, as a special kind of the pro-form, is used. The conclusion points out that pro-forms can be employed as long as they do not interfere with the continuity of topic and the cohesion of text.

\section{PREMESSA}

La linguistica testuale tenta da una ventina d'anni di definire i meccanismi di coesione, di costruzione, di organizzazione e progressione di un testo sul piano della forma e della coerenza interna che servono così a mostrare una generale strategia di massima esplicitezza del testo.

In questo articolo vogliamo indagare uno di questi meccanismi, cioè la sostituzione sintagmica ${ }^{1}$ ossia l'uso di certe pro-forme ${ }^{2}$ nella rete formale-semantica di un testo. Ci addentreremo quindi in uno dei fenomeni di referenza testuale che a volte presentano a chi genera ed interpreta i testi problemi di dimensionalità, di concordanza, d'assenza di rimando, di non adeguatezza ecc. Chi produce un testo dovrebbe padroneggiare e rendersi conto del valore di certi mezzi di coreferenza in

${ }^{1}$ Cf. Verlato, 1995, p. 52, che cita R. Harweg, Pronomina and Textkonstitution, Fink, München 1968, p. 20: "Sostituzione è la sostituzione di un'espressione linguistica con un'altra espressione linguistica determinata. La prima di queste due espressioni quella sostituita o da sostituire, si chiama Substituendum, la seconda, quella che sostituisce, Substituens".

${ }^{2}$ „Pro-forme [...] sono tutte quelle espressioni con le quali un parlante può far riferimento ad oggetti e stati di cose, ai quali egli ha già fatto riferimento mediante un antecedente nel pre-testo", Conte, 1988, p. 21. 
quanto segni che tracciano la coesione del testo. La coreferenza, la intendiamo in questa sede come una relazione simmetrica di referenza tra due o più termini in un testo (cf. Adam, 1990, p. 52).

\section{SOSTITUZIONE PRONOMINALE}

Una prima proprietà rilevante di coreferenza è il fenomeno di pronominalizzazione. In modo uguale se ne servono la lingua scritta e il discorso parlato. Alcuni linguisti trattano il fenomeno della pronominalizzazione perfino „,come mezzo principale, [...], della costituzione del testo" (Verlato, 1995, p. 51).

All'origine i pronomi sono forme semanticamente quasi vuote. Secondo de Beaugrande e Dressler (1984, p. 90) sono le pro-forme più note quelle „che adempiono la funzione di sostantivi o sintagmi nominali con i quali essi coriferiscono". Così si potrebbe spiegare l'etimologia del termine di pronome < pronome. Ciò non toglie che una tale proforma possa sostituire, come vedremo nel seguito di questo articolo, diverse parti del discorso oppure interi enunciati. Osserviamo che i pronomi sostituenti vengono interpretati perché si riconosce la situazione in cui l'atto comunicativo si svolge, e, soprattutto, perché se li riferisce ad un individuo menzionato nel testo attraverso una epressione linguistica.

I pronomi impiegati nel procedimento di sostituzione sono prima di tutto: a) i pronomi personali di terza persona tonici: egli, ella, loro, lui, lei, esso, essa, essi, esse; b) i pronomi atoni diretti di terza persona: $l a, l o, l i, l e$; c) il pronome $n e$; d) i pronomi dimostrativi: questo, ..., quello, ..., ciò; e) i pronomi possessivi. il mio, ...; f) i pronomi indefiniti: un altro, qualcuno, ...

Svolgendo la loro funzione particolare, questi pronomi con altre forme sostitutive, alcune delle quali vedremo negli esempi seguenti, stabiliscono una rete di legami testuali che danno coesione e coerenza al testo.

Dal punto di vista semantico e pragmatico, il pronome viene caricato dal senso e dall'aspetto dell'oggetto a cui si riferisce nelle realtà contestuale e co-testuale ${ }^{3}$.

Prendiamo in esame i testi:

\{1\} Perchě, novizio che si avviava ai misteri del santo sacerdozio di Dio, anch'io umile fanciullo compresi che l'Abate sapeva qualcosa ma lo aveva appreso sotto il sigillo della confessione. Egli doveva aver saputo dalle labbra di qualcuno qualche particolare peccaminoso che poteva avere relazione con la tragica fine di Adelmo.

(Eco, 1980, Antol.: p. 673)

\{2\} La prima volta che ho visto i capelloni, è stato a Praga. Nella hall dell'albergo dove alloggiavo sono entrati due giovani stranieri, con $i$ capelli lunghi fino alle spalle. Sono passati attraverso la hall, hanno raggiunto un angolo un po' appartato e si sono seduti a un tavolo. Sono rimasti lì seduti per una mezzoretta, osservati dai clienti,

${ }^{3}$, Co-testo = regolarità interne al testo; contesto = condizioni, esterne al testo della produzione e della ricezione testuale", Verlato, op. cit., p. 28. 
tra cui io; poi se ne sono andati. Sia passando attraverso la gente ammassata nella hall, sia stando seduti nel loro angolo appartato, i due non hanno detto parola [...].

$E s s i$, infatti, in quella particolare situazione - che era del tutto pubblica, o sociale, e starei per dire, ufficiale - non avevano affatto bisogno di parlare.

(Pasolini, 1975, p. 9)

Il pronome egli in $\{1\}$ coriferisce con l'Abate; è quindi la ripresa pià corta del nome e del suo significato appreso attraverso la lettura del testo. Il significato proprio del pronome egli è molto di meno esteso: il valore grammaticale maschile-singolare e una sola accezione, tratto umano.

Per quanto riguarda $\{2\}$, la pro-forma essi (il plurale di egli, riferito ora ad oggetti ora a persone) corrisponde a due giovani stranieri e tutto quanto che apprendiamo su di loro.

In entrambi i casi il pronome concorda in numero, genere e persona con il suo referente; ciò vale pure per tutti i pronomi usati in sostituzione.

\section{PRONOMINALIZZAZIONE ANAFORICA E CATAFORICA}

I pronomi che fungono da substituentia rinviano a referenti già dati o che saranno detti. Conte $(1988$, p. 22) li qualifica come riprese sintattiche che sul piano paradigmatico corrispondono ad un antecedente nel testo. Il rinvio così stabilito è in sostanza una sostituzione che avrà sia carattere anaforico, sia quello cataforico.

Conte (1992, p. 27) ritiene che: „Sono anafore quelle espressioni con le quali si fa riferimento a una entità alla quale si è già fatto riferimento con un antecedente nel co-testo precedente (o che è già presente nell'universo di discorso)". La definizione qui sopra potrebbe essere applicata pure alla catafora, ma con la differenza che in caso di anafora una pro-forma si mette dopo l'espressione coreferente, mentre in caso di catafora, la pro-forma si trova prima.

Vediamo tre esempi:

(3) La sottocultura al potere ha assorbito la sottocultura all'opposizione e l'ha fatta propria: con diabolica abilità ne ha fatto pazientemente una moda, [...].

(Pasolini, 1975, p. 13)

\{4\} Non è questa la sede per indicare gli obiettivi e il senso della riforma goldoniana, inserita in quel vasto moto di rinnovamento della cultura occidentale che va sotto il nome di Illuminismo.

(Bertocchi e al., Antol.: p. 952)

\{5\} E' più che giusto: e lei ha assicurato a sua figlia un avvenire di ricchezza... Ma non so se sua figlia riuscirebbe a giustificare quel che lei ha fatto per assicuragliela, questa ricchezza...

(Sciascia, 1971, Antol.: p. 657)

I pronomi (anaforici) la e ne in $\{3\}$ coriferiscono al sintagma nominale la sottocultura all'opposizione che è il loro antecedente. In $\{4\}$, invece, il pronome questa è coreferente con il sostantivo la sede; ciò instaura la sostituzione cataforica 
tra essi. L'elemento (la sede) che segue la pro-forma cataforica (questa), lo chiameremmo in questa sede susseguente.

Notiamo un caso particolare in $\{5\}$ : il pronome la rimanda ad un elemento (ricchezza) che lo precede nel testo e ad un altro (questa ricchezza) che lo segue. Abbiamo dunque qui a che fare con una doppia funzione di pro-forma: anaforica e cataforica allo stesso tempo. Essa pare dovuta alla ripresa lessicale dimostrativa (cf. Adam, op. cit., p. 55-61) del termine di ricchezza.

Il rivelare di anafore o di catafore nel testo può generare problemi, se si ha un troppo lungo tratto testuale dopo o prima dell'apparire della pro-forma. Le espressioni coreferenti vengono in genere usate entro un singolo enunciato o gruppi di due - tre frasi.

Osserviamo l'esempio:

[6\} Alla Mora ci stava già Nicoletto, e Santa (a), che non poteva soffrirlo, appena morta la madre era scappata a Canelli, s'era presa una stanza, e aveva fatto la maestra (b). Ma col tipo che lei (c) era, aveva subito trovato da impiegarsi alla Casa del fascio, e dicevano di un ufficiale della milizia, dicevano di un podestà, del segretario, dicevano di tutti i più delinquenti là intorno. Cosi bionda, cosi fina $(d)$, era il suo posto salire in automobile e girare la provincia, andare a cena nelle ville, nelle case dei signori, alle terme d'Acqui - non fosse stata quella compagnia. Nuto cercava di non vederla $(e)$ per le strade, ma passando sotto le sue finestre alzava gli occhi alle tendine.

(Pavese, 1984, Antol.: p. 631)

Consideriamo che la distanza entro la pro-forma la e il nome Santa è assai lunga e sarebbe astruso da rintracciare la coreferenza anaforica tra questi elementi se non ci fossero altri referenti di Santa (nome proprio di una donna) nella sequenza di frasi: una pseudo-ripresa lessicale (b), il pronome personale tonico (c), una specificazione (d).

\section{CATENE COREFERENZIALI}

La successione (a) - (b) - (c) - (d) in \{6\} è par excellence una catena di referenti anaforici che risponde alle esigenze definite da Berretta (1990, p. 95): ,$\ldots$ un antecedente difficile da recuperare, per es. Iontano nel testo, in concorrenza con altri antecedenti potenziali etc. [...] dovrà essere richiamato con una proforma ben esplicita, poniamo un pronome tonico, o un dimostrativo, o una proforma lessicale, eventualmente inserita in una struttura sintattica a sua volta marcata: insomma, un elemento che porti su di sé tratti dell'antecedente sufficienti, in quell'intorno testuale, ad identificarlo, oltre che a segnalare, per sua propria forma esplicita e per l'eventuale struttura sintattica marcata che lo contiene, il fatto che la ripresa voluta è difficile".

L'identificazione dell'oggetto nella catena anaforica sembra un processo graduale in cui gli elementi succedenti al nome iniziale (cf. Dobrzyńska, 1993, 
p. 28) sono i suoi substituentia che vanno dal valore derivazionale più specifico (sinonimi, iperonimi, classi generali, epiteti) a quello meno determinato (pronomi).

Al pronome, in tal caso, gli attribuiremo il ruolo riduzionale decodificato in presenza di antecedenti diretti o indiretti. Ciò non significa però che il pronome „riduca" il senso del suo referente. Anzi, comprende tutte le informazioni aggiunte dai nomi derivazionali nella catena anaforica. Così contribuisce alla ,compattezza $\mathrm{e}$ la chiarezza dell'enuciazione" (de Beaugrande e Dressler, op. cit. p. 96).

Meno sovente è l'uso della catafora nella catena di riprese coreferenziali. Nell'esempio seguente il pronome indefinito qualcun altro è una ripresa cataforica del referente Kevin il quale è seguito da tre riprese anaforiche:

(7) C'era anche qualcun altro (1) che stava sognando. Nella mansarda dei McCallister, Kevin (2) si agitò girandosi da una parte all'altra del letto con la visione di tutti quei pezzi di cadavere nascosti nel seminterrato del vecchio Marley. Braccia, gambe e teste gettate qua $\mathrm{e}$ là con i vermi $\mathrm{e} i$ topi che banchettavano...

Il terrore lo (3) fece sobbalzare costringendolo (4) a svegliarsi. Si passò una mano sugli occhi e si guardò intorno. Per una volta era contento (5) di trovarsi nella soffitta.

(Mamma, ho perso l'aereo, p. 23)

La catena di legamenti coreferenziali di questo brano si presenterebbe così:

la proforma cataforica (1)

$\kappa$

$\frac{\text { contenuto di riprese (2) }}{y}$

la proforma anaforica (3)

$\searrow$ la proforma anaforica (4)

$\checkmark$ la specificazione anaforica (5)

L'uso della catafora in una tale catena produce un effetto stilistico idoneo a non rivela immediatamente l'identità del referente.

\section{CLITICI ED ALTRE PRO-FORME ENUNCIATIVE}

Come abbiamo ormai sottolineato l'applicazione di una pro-forma concerne non solo questi referenti che sono nomi o sintagmi nominali. Si è verificato che $i$ pronomi atoni $l o$, ne e i pronomi dimostrativi questo, quello, ciò possono sostituire anaforicamente o cataforicamente un enunciato / una frase o una sequenza di enunciati / frasi. (cf. Matronola e Tadiello, 1986, p. 42) Diamo uno sguardo agli esempi:

\{8\} „Tanto vale che te $\boldsymbol{l o}(1)$ dica" fece Nuto d'improvviso senza levare gli occhi, „io so come l'hanno ammazzata. C'ero anch'io"(2)

(Pavese, 1984, Antol.: p. 631)

[ripresa pronominale cataforica: pro-forma (1) $\Leftarrow \underline{\text { enunciato (2)] }}$ 
\{9\} Non sono rimasto imbarazzato (1) e lo (2) ho deplorato, non soltanto per il rapporto fra il presidente e Monica, ma anche per il modo con cui è stato trattato, l'eccessiva enfasi posta su di esso, la trivializzazione dei suoi aspetti politico e giudiziario.

(„Corriere della Sera”, 24 settembre 1998, anno 123, n. 226, p. 11)

[ripresa pronominale anaforica: enunciato (1) $\Rightarrow$ pro-forma (2)]

$\{10\}$ Da oggi in poi lasciami perdere. Non annoiarmi con le concioni, $i$ deliri o le analisi su questa situazione. (1). Monica, non ne (2) posso più.

(L'Espresso, N. 41, anno XLIV, 15 ottobre 1998, p. 221)

[ripresa pronominale anaforica: enunciato (1) $\Rightarrow$ pro-forma (2)]

\{11\} Ciò che (1) sostituiva il tradizionale linguaggio verbale, renendolo superfluo - e trovando del resto immediata collocazione nell'ampio dominio dei „segni", nell'ambito cioè della semiologia - era il linguaggio dei loro capelli (2).

(Pasolini, 1975, p. 9)

[ripresa pronominale cataforica: proforma (1) $\Leftarrow$ enunciato (2)]

Abbiamo inoltre constatato che degli interi enuciati, frammenti di testo o discorso vengono sostituiti da altre strutture linguistiche pro-forma come: la cosa, tutto questo, qualcosa, tutte queste cose, tutto quanto ecc. Gli attribuiremmo il nome di pro-forme enuciative. Vediamo due esempi:

\{12\} [...] per la religione e soprattutto per la Chiesa non c'è più spazio. La lotta repressiva che il nuovo capitalismo combatte ancora per mezzo della Chiesa è una lotta ritardata, destinata nella logica borghese, a essere ben presto vinta, con la conseguente dissoluzione „naturale” della Chiesa (1). Sembra folle, ripeto, ma il caso dei jeans „Jesus" è una spia di tutto questo (2). Coloro che hanno prodotto questi jeans e li hanno lanciati nel mercato, usando, per lo slogan di prammatica uno dei dieci Comandamenti dimostrano [...] di essere già oltre la soglia entro cui si dispone la nostra forma di vita e il nostro orizzonte mentale.

(Pasolini, 1975, pp. 16-17)

$$
\text { [ripresa anaforica: enunciato (1) } \Rightarrow \text { pro-forma (2)] }
$$

(13\} Kevin vagabondò un po' per lo studio. Sapeva che cosa (1) sarebbe accaduto di lì a poco. Avrebbe dovuto trascorrere la sua prima notte tutto solo (2).

(Mamma, ho perso l'aereo, p. 38)

[ripresa cataforica: pro-forma cataforica (1) $\Leftarrow$ enunciato (2)]

\section{PRO-FORME E COMPATTEZZA STILISTICA}

Si rivela che l'inserire delle pro-forme nel testo può essere un procedimento stilistico di scelta (ad es., per evitare la ripetizione, cf. $\{14\}$ ) o una necessità d'uso (per eliminare l'ambiguità, cf. $\{15\}$ ). Osserviamolo prendendo sempre in considerazione il meccanismo di pronominalizzazione: 
\{14\} [...] le faccio vedere quel che ha scritto il Dibella poche ore prima di morire - gli mostrò la copia fotografica della lettera.

Don Mariano la prese e la guardò allontanandola per tutta la lunghezza del braccio. Disse che vedeva bene le cose lontane.

- Che gliene pare? - domandò il capitano.

(Sciascia, 1971, Antol.: p.656)

\{15\} Nuto giocava con delle pietruzze e guardò in su. „Non vuoi che andiamo a Gaminella in alto? Andiamoci. E' presto"' Allora partimmo, e lui si mise avanti per i sentieri delle vigne.

(Pavese, 1984, Antol.: p. 630)

In $\{14\}$ il pronome la, nonché ne, li identifichiamo con il sintagma la copia fotografica della lettera: il procedimento rende il testo più agevole. Sarebbe superflua e scorretta la versione.

[*] l...] gli mostrò la copia fotografica.

Don Mariano prese la copia fotografica della lettera e guardò la copia fotografica della lettera allontanando la copia fotografica della lettera. Disse che vedeva le cose lontane.

- Che le pare della copia fotografica della lettera? - domandò il capitano

Un testo così costruito non risponderebbe alle norme di composizione testuale e particolarmente alla „méta-règle de répétition” stabilita da Charolles ${ }^{4}$.

In $\{15\}$, il pronome lui avente per il suo antecedente $N u t o$, va d'accordo con le regole di ripetizione e un'altra „měta-rěgle” di Charolles, ossia quella di „relation”.

Vediamo che effetto darebbe il tralasciamento del pronome lui (supplementare con la forma verbale) in $\{15\}$ :

[**] Nuto giocava con delle pietruzze e guardò in su. „Non vuoi che andiamo a Gaminella in alto? Andiamoci! E' presto".

Allora andammo, e si mise avanti per $i$ sentieri delle vigne.

In effetti, non si sa più chi „si mise avanti per $i$ sentieri delle vigne"; non esiste più il rapporto corelazionale fra Nuto e il soggetto dell'azione: la comprensione del testo si dimostra compromessa.

Qualche volta l'antecedente del pronome nel testo non è individuabile. Esaminiamo l'esempio:

$\{16\}$ - Non faccio affari: vivo di rendita.

- Che rendita?

- Terre e boschi.

- Quanti ettari ne possiede?

(Sciascia, 1971, Antol.: p. 656)

4 «Pour qu'un texte soit [...] cohérent, il faut qu'il comporte dans son développement linéaire des élèments à récourence stricte», Charolles, 1978, p. 14.

5 «Pour qu'une séquence ou un texte soient cohérents, il faut que les faits qu'il dénote dans le monde représenté soient reliés», ibid. p. 31. 
Vi è evidente l'ambiguità di coreferenza: la particella ne sostituisce sia terre sia boschi sia ambedue i termini. La decodificazione del messaggio rimane incerta malgrado l'accettabilità di ogni opportunità.

Non di rado, per evitare il rischio d'ambiguità risultante a un'eventuale pronominalizzazione, è indispensabile ricorrere all'uso di una ripetizione. Consideriamo la seguente sequenza di frasi:

\{17\} La commedia (17-1) si apre subito con una azione (17-2) scenica; tutte le notizie sui personaggi, sull'antefatto, sul movente dell'azione (17-3) e i suoi possibili sviluppi si ricavano dal dialogo dei personaggi.

Possiamo anzi dire che la commedia (17-4) dell'avaro comincia solo alla scena (17-5) III, che corrisponde all'inizio dell'Aulularia, mentre le scene (17-6) I e II assolvono alla funzione svolta nella commedia (17-7) latina dal prologo.

(Bertocchi e al., 1994, p. 950)

La soppressione della ripetizione in $\{17\}$ potrebbe provocare del disturbo nella comprensione generale del brano e in totale nella prospettiva funzionale del testo. Vediamo che esito dà la pronominalizzazione in questo esempio:

[ $^{* * *}$ I La commedia si apre con una azione scenica; tutte le notizie sui personaggi, sull'antefatto, sul movente di essa (i) e $i$ suoi possibili sviluppi si ricavano dal dialogo dei personaggi. Possiamo anzi dire che quella (ii) dell'avaro comincia solo alla scena III, che corrisponde all'inizio dell'Auluraria, mentre quelle (iii) I e II assolvono alla funzione svolta in quella (iv) latina dal prologo.

Diversi fattori decidono che la sostituzione tramite le proforme essa, quella, quelle in $\{17\}$ non è possibile:

a) i termini potenzialmente sostitutivi, commedia, azione, scena, sono del genere femminile e i pronomi proposti al posto di essi suscitano confusione: se trattassimo le pro-forme come riprese anaforiche sintattiche essa (i) e quella (ii) costituirebbero anafore di commedia ovvero di azione, invece quelle (iii) e quella (iv) assumerebbero ancora un altro referente, scena. Ciò si rivela assurdo e non accettabile.

b) Quella (ii) e quella (iv) non potrebbero perfino essere riprese semanticamente o pragmaticamente di commedia (17-1): non esiste equivocità tra (17-1), (17-4) e (17-7): il significato di (17-1) è: composizione destinata alla rappresentazione scenica, il signifiato di (17-4): azione, buffonata dell'avaro, il significato di (17-7): genere letterario di una specifica caratteristica.

c) il pronome quelle (iii) coreferente in [***] con scene (17-6) non dà possibilità di una decodificazione corretta malgrado l'univocità di (17-5) e (17-6) come pure la loro ,vicinanza” nel testo.

d) azione (17-3) sembra ormai un'anafora di azione (17-2) perché comporta un determinante (articolo determinativo) che segnala il rapporto intrinseco tra (17-3) e $(17-2)$. 


\section{RINOMINALIZZAZIONE E ALTRE PRO-FORME CATEGORIALI}

A questo punto constatiamo che la pronominalizzazione in [***] condurrebbe a uno scompiglio nell'interpretazione del testo. Quindi, la ripetizione in certi casi può, a fortiori, avere aspetto di pro-forma e così fare da mezzo di coerenza testuale. Per distinguerlo dalla ripetizione vista come un errore contro la „méta-rěgle de répétition" questo procedimento viene chiamato nella linguistica testuale la rinominalizzazione (cf. Conte, 1988, p. 22).

Alcuni linguisti ritengono che la rinominalizzazione svolga diverse funzioni stilistiche ad es.: demarcazione che delimita sezioni del testo, centralizzazione di idee, stabilità ed esattezza del contenuto ecc.

La si usa spesso in testi di linguaggi specializzati, per es. matematici, filosofici, tecnici.

Si veda un frammento di un manuale tecnico per la scuola media:

\{18\} La presa è il punto in cui si attacca, tramite la sua spina, un utilizzatore qualsiasi. A differenza degli interruttori, che servono ad aprire o chiudere un circuito elettrico, le prese sono sempre in tensione, in esse passa sempre la corrente. Una presa presenta generalmente tre fori. Può accadere che l'utilizzatore, dopo aver inserito la spina, non funzioni; se collegandolo ad un'altra presa esso funziona regolarmente, significa che la presa precedente non è più in tensione. In questo caso si stacca innanzitutto l'interruttore centrale, isolando così l'impianto, e poi si svitano le viti che tengono attaccata alla parete la piastrina che copre la presa.

(Luisi e Luisi, 1983, p. 346)

Il fenomeno di rinominalizzazione viene spesso riscontrato nel discorso parlato:

\{19\} (dialogo tra due camionisti)

- Mi disse pure ch'è un buon rimedio.

- Un buon rimedio?

- Un buon rimedio. Oltretutto è un buon rimedio, disse.

- Anche sposarsi è un buon rimedio.

- Io sono già sposato.

- Io mi sposo domani.

(Vittorini, 1980, Antol.: p. 605)

De Beaugrande e Dressler $(1984$, p. 82) chiamano il meccanismo riscontrato in $\{18\},\{19\}$ la ricorrenza. Secondo questi linguisti ,la ricorrenza è frequente quando si parla in modo spontaneo perchě si ha poco tempo a disposizione per pianificare l'enunciazione e perché il testo di superficie si disperde rapidamente". Gli autori citano alcuni fattori contestuali che producono la ricorrenza. Ne ritroviamo in \{19\}: rafforzare e sottolineare il proprio parere; la sorpresa per fatti che sembrano essere in disaccordo col proprio punto di vista, il rifiuto, l'esplicitazione.

La ricorrenza di cui parlano de Beaugrande e Dressler è, certo, di carattere lessicale. Loro ammettono che qualche volta la ricorrenza possa essere parziale 
(ibid., p. 85); la si determina così quando si usa lo stesso materiale linguistico cambiando genere di parola (cf. (17-5) e (17-6)).

Elementi che possono esser messi in relazione con delle pro-forme non sono solamente i nomi, i sintagmi nominali o gli enunciati. Per rendere il contenuto di un verbo o sintagma verbale si ricorre a volte alluso del verbo fare che in questa sede va chiamato pro-verbo.

$\{20\}$ Chi non ha mai impaginato deve sapere che per stabilire quanto verrà grande una immagine bisogna prendere l'originale, cicoscriverne la porzione da riprodurre, tracciare una diagonale, poi prolungare idealmente o fisicamente quella diagonale sul foglio d'impaginazione ( 1 ), secondo la grandezza che si desidera per l'immagine, eccetera eccetera. Munari faceva (2) tutto in un secondo, oggi si direbbe virtualmente, a occhio, posava la matita sul foglio e la pagina era già perfetta (3).

(Eco, U., L'Espresso, N. 41, anno XLIV, 15 ottobre 1998, p. 278)

In $\{20\}$ registriamo una doppia funzione referenziale del pro-verbo fare (2): sostituisce quanto dislocato a sinistra ed a destra di esso in una ripresa anaforico-cataforica:

$$
\underline{\text { referenti verbali (1) } \Rightarrow \underline{\text { pro-verbo (2) }} \Leftarrow \text { referenti verbali (3) }}
$$

Dobbiamo dire che nell'elenco dei mezzi lessico-sintattici sostitutivi, oltre alla pronominalizzazione anaforica e cataforica, le riprese derivate, la rinominalizzazione, il pro-verbo, non sono da escludere ancora possibilità di stabilire delle pro-forme di tali categorie grammaticali come avverbi, aggettivi o altri elementi linguistici presenti nel testo.

Ad esempio, consideriamo il pseudo-pronome "lo" come una pro-forma (in sostanza anaforica) dell'attributo nella sequenza:

(21) Il loro silenzio era rigorosamente funzionale. E lo era semplicemente, perché la parola era superflua.

(Pasolini, 1975, p. 9)

Opure l'avverbio laggiù come una pro-forma cataforica del complemento di luogo al convento in $\{22\}$ :

\{22\} [...] ti voglio bene cento volte di più adesso che son lontana da te e che non ti ho più accanto ad ogni ora del giorno come laggiù, al convento.

(Verga, 1993, p. 22)

\section{CONCLUSIONI}

Quasi ogni volta quando si distinguono delle pro-forme nel testo, i loro antecedenti sono recurabili nel medesimo testo. Rari sono i casi in cui l'antecedente è difficile da delineare o perfino sottinteso. Vediamo un brano in cui l'antecedente del pronome ne non ha il suo referente proprio nel testo; lo si capisce attraverso 
l'uso del pronome quantitativo tanti che segnala un'ellissi dell'antecedente inteso allora come: individui, uomini, soldati, nemici ...

\{23\} Quando ci fu il rastrellamento di giugno e per quei sentieri ne morirono tanti, Santa si difese tutta una notte con Baracca in una cascina dietro Superga.

(Pavese, 1984, Antol.: p. 633)

Come abbiamo osservato in diversi esempi messi in esame in questo articolo, la pronominalizzazione anaforica o cataforica è il mezzo più frequente ed efficace (se segue le regole di pertinenza) del meccanismo di referenza testuale. I pronomi, non dotati di significato in sè - se non di quello grammaticale - non forniscono autonomamente vere e proprie informazioni; servono al compito di segnalare dove, all'interno del testo, le informazioni vanno cercate. Così, come segnali di recupero di elementi presenti nel testo, contribuiscono a creare una rete di legami che danno un testo coeso e coerente.

Affinché un tale scopo sia soddisfatto, i pronomi e altre pro-forme definite devono obbedire sempre a qualche principio generale, ad es.

- quello della inequivocità che dà coesione e coerenza testuale ,qualora la coreferenza non sia ambigua, qualora cioè l'elemento linguistico che viene ripreso sia chiaramente individuabile" (Matranola e Tadiello, 1986, p. 43),

- quello della economicità che risponde alla esigenza generale di economia nella produzione verbale (cf. esempio \{3\}) come sostengono de Beaugrande e Dressler (1984, p. 97): „La pro-forma riduce lo sforzo elaborativo perché è più breve dell'espressione che sostituisce".

- quello della variazione che permette di variare mediante una sostituzione a fine di togliere una ripetizione,

- quello del buon senso che rende il testo più agevole per chi lo interpreta: si è accertato, per es., che non sempre la ripetizione sia un mezzo stilistico erroneamente utilizzato. Anzi, costituisce spesso un fattore rilevante della coesione e coerenza.

Il principio del buon senso ha pure il suo rilievo nella formulazione delle proforme lessicali derivate in una catena anaforica: secondo alcuni linguisti il termine derivato non dovrebbe essere semanticamente più ricco del suo referente (cf. Dobrzyńska, 1993, p. 29); d'altra parte, l'allargamento del contenuto referenziale può generare nuove catene di legamenti che non devono per forza essere contro il seguente principio:

- cioè, quello di continuità tematica che bada a non cambiare topic nel testo (cf. Conte, 1988, pp. 50),

- quello di compattezza stilistica che stabilisce una posizione possibile della pro-forma nell'ordine lineare di elementi in relazione anaforica o cataforica.

Badando a queste restrizioni e tenendo conto della loro varietà si riesce ad evitare ogni indeterminatezza dell'antecedente di una pro-forma, il che favorisce la comprensione e l'interpretazione di un testo. 
L'approccio di alcuni procedimenti di sostituzione coreferenziale esposti in questo articolo non è di sicuro esausto - ciò non era il nostro scopo -, ma speriamo che le osservazioni ivi delineate innalzino l'idea menzionata da molti linguisti di creare una "grammatica del testo" che serva a chi insegna basandosi sui testi, si butta nella loro correzione ovvero nell'interpretazione dei testi come atti intuitivi di comunicazione e fonte di messaggi.

\section{BIBLIOGRAFIA}

A d a m J.-M., Eléments de linguistique textuelle, Mardaga, Liège 1990.

Beaugrande de, R.-A. Dressler W.U., Introduzione alla linguistica testuale, il Mulino, Bologna, traduzione di S. Muscas, 1984; ed. orig.: Einführung in die Textlinguistik, Max Niemeyer Verlag, Tübingen 1981.

Berretta M., Riprese anaforiche e tipi di testo: il monologo espositivo, Parallela 2, Lichen K. e al., Tübingen, 1986.

Berretta M., Catene anaforiche in prospettiva funzionale: antecedenti difficili, Rivista di Linguistica, 2,1, 1990.

Charolles M. „Introduction aux problèmes de la cohèrence des textes”, in: Langue française 38, Larousse, Paris 1978.

Conte M.-E., Condiziomi di coerenza. Ricerche di linguistica testuale, Pubblicazioni della Facoltà di Lettere e Filosofia dell'Università di Pavia 46, La Nuova Italia, Firenze 1988.

Conte M.-E., Anafore nella dinamica testuale, in: La centralità del testo nelle pratiche didattiche, P. Desideri, (a cura di), Quaderni del Giscel, 196/10, 1992.

Dobrzyńska T., Tekst. Próba syntezy, IBL, Warszawa 1993.

Matranola M. e Tadiello S., La sostituzione in forma pronominale, in: Prospettive didattiche della linguistica del testo, La Nuova Italia, Firenze, 1986.

Pohl E., La linguistica testuale in ltalia: ricezione, sviluppi, studi applicati, in: Studi italiani di linguistica teorica e applicata. A. XIV, n. 1-3, 1985.

Reboul A. e Moeschler J., Pragmatique du discours, Armand Colin, Paris 1998.

Renzi L., Grande grammatica italiana di consultazione, l. La frase. I sintagmi nominale $e$ preposizionale, il Mulino, Bologna 1991.

Verla to M., Avviamento alla linguistica del testo, UNIPRESS, Padova 1995.

\section{TESTI ANALIZZATI}

Bertocchi D. e al., Antologia italiana per il biennio, La Nuova Italia, Firenze 1994, in cui frammenti trati di:

Eco U., Il nome della rosa, Bompiani, Milano 1980.

Pavese C., La luna e i falò, Mondadori, Milano 1984.

Sciascia L., ll giorno della civetta, Einaudi, Torino 1971.

Vittorini E., Uomini e no, Mondadori, Milano 1980.

Inoltre:

Mamma, ho perso l'aereo, basato sulla sceneggiatura di J. Hughes, La biblioteca di „Noi”, 1993.

Luisi M. e Luisi R., Pensiero e tecnica, SEI, Torino 1985.

Verga G., Storia di una capinera, TEN, Roma 1993.

Pasolini P.P., Scritti corsari, Garzanti, Roma 1975. 